\title{
Enhanced Effective Thickness of Multi-Layered Laminated
}

\section{Glass}

\author{
LAURA GALUPPI \\ Gianni Royer-Carfagni \\ Department of Industrial Engineering, University of Parma \\ Parco Area delle Scienze 181/A, I 43100 Parma, Italy \\ tel: +39-0521-905917, fax: +39-0521-905924, email: gianni.royer@unipr.it
}

\begin{abstract}
The stiffness and strength of laminated glass, a composite of glass layers bonded together by polymeric interlayers, depend upon shear coupling between the glass plies through the polymer. In the design practice, this effect is commonly considered by defining the effective thickness, i.e., the thickness of a monolith with equivalent bending properties. Traditional formulations have been proposed for a package of two layers of glass and one polymeric interlayer, but their extrapolation to a higher number of layers gives in general inaccurate results. Here, the recently-proposed Enhanced Effective Thickness method is extended to the case of laminated glass beams composed $i$ ) by three layers of glass of arbitrary thickness, or ii) by an arbitrary number of equally-thick glass layers. Comparison with numerical experiments confirms the accuracy of the proposed approach.
\end{abstract}


KEYWORDS: multi-layered composites, multi laminated glass, safety glass, effective thickness, polymeric interlayer.

\section{Introduction}

Laminated glass is a composite widely used in civil engineering, as well as in automotive, aeronautics and shipbuilding. It is typically made of two glass plies bonded by a thermoplastic polymeric interlayer with a treatment in autoclave at high pressure and temperature. This process induces a strong chemical bond between materials, due to the union between hydroxyl groups along the polymer and silanol groups on the glass surface. Through lamination, safety in the post-glass-breakage phase is increased because fragments remain attached to the interlayer; risk of injuries is reduced and broken glass maintains a certain cohesion that prevents catastrophic collapse.

In the pre-glass-breakage phase, the polymeric interlayers are too soft to present flexural stiffness per se, but they can provide shear stresses that constrain the relative sliding of the glass plies ([1]). The precise calculation of this coupling is quite difficult and usually requires numerical analysis, complicated by the fact that response of the polymer is nonlinear, viscoelastic and temperature dependent. A common practice is to consider the polymer as linear elastic accounting for its viscoelasticity through an equivalent elastic modulus, assumed equal to the relaxed modulus under constant strain after a time comparable to the duration of the design action. Of course, the degree of coupling of the glass layers depends upon the shear stiffness of the interlayer ([2]). Thus, the flexural performance is somehow intermediate between the two borderline cases ([3], [4]) of $i$ ) monolithic limit, with perfect bonding between glass plies (shear-rigid interlayers) and ii) layered limit, with frictionless sliding glass plies. Since stress and strain are much lower in the monolithic than in the layered limit, to avoid redundant design a large number of studies, also in recent years, 
have considered this subject $([5,6,7])$.

In numerical computations, laminated glass may be modeled with layered shell elements that take into account the competing stiffness between glass and interlayer, but the libraries of most commercial numerical codes do not contain such elements; on the other hand, a full three-dimensional analysis is complicated and time consuming. This is why, in the practice and especially in the preliminary design, it is useful to rely upon simple methods. The most common approach consists in defining the so called effective thickness, i.e., the thickness of a glass monolith with bending properties equivalent to the laminated element. More precisely, the effective thickness of a laminated glass plate is the (constant) thickness of a monolithic plate that, under the same boundary and load conditions, presents the same maximal stress or maximal deflection. This is a very practical definition, but the literature and the technical standards record various conflicting formulas for its quantification.

The most used formulations are the one prescribed by the European Project Norm prEN13474 [8] and that recorded in ASTM E1300 [9] following the proposal by Bennison et al. $[10,11]$ and the original work by Wölfel [12]. The first formulation condenses all the effects of the interlayer in one coefficient $\omega$ provinding a linear interpolation between the layered and the monolithic limit; however, as it will be demonstrated later on in Section 4.2, this method is quite crude and leads to contradictory results. On the other hand the method of ASTM E1300, as discussed in $[13,14]$, gives excellent results for laminated beams under uniformly distributed loading, but is inaccurate in other cases. Very recently, an alternative method [13], called Enhanced Effective Thickness, has been proposed by the authors. This is based upon a variational approach where, through minimization of the strain energy functional, the best approximation for the response of laminated glass is selected among a restricted class of shape functions for the deflection surface. The main underlying hypotheses are: i) the interlayer has no axial or bending stiffness, but only shear stiffness; ii) shear strain of glass is negligible; iii) both glass and polymer are linear elastic materials; iv) geometric 
non-linearities are ineffective. Remarkably the method, originally conceived of for beams under bending [13], can be naturally extended to the two-dimensional case of plates [14] under the most various load and boundary conditions.

In general, all the aforementioned methods have been formulated for laminates with two glass layers and one interlayer. Attempts have been made to extend the prEN-13474 and the ASTM E1300 methods to the case of three of more glass plies, but the accuracy is in general not satisfactory, as it will be shown later on in Section 4. The purpose of this Article is to show that the Enhanced Effective Thickness method can be naturally extended to the case of multilayered laminated glass beams. More specifically, the cases that will be treated here are laminated beams composed either by three glass layers of arbitrary thickness, or by an arbitrary number of equally-thick glass layers. By defining an effective moment of inertia of the composed beam as the weighted harmonic mean of the moments of inertia corresponding to the layered and monolithic limit, practical formulas for the stressand deflection-effective thickness are proposed. The method covers various boundary and loading conditions. Comparisons with numerical experiments highlight the much higher accuracy of the proposed approach with respect to the other formulations.

\section{Five-layered beams with glass plies of arbitrary thickness.}

With respect to a reference system $(x, y)$ as in Fig. 1, consider the laminated beam of length $l$ and width $b$, composed of three glass plies, of thickness $h_{1}, h_{2}$ and $h_{3}$ and Young's mudulus $E$, bonded by thin polymeric interlayers (of thickness $t_{1}$ and $t_{2}$ respectively), whose shear modulus is denoted by $G$. The beam is loaded by an arbitrary load per unit length $p(x)$, 
not necessarily uniformly distributed. Let

$$
A_{i}=h_{i} b, I_{i}=\frac{b h_{i}^{3}}{12}(i=1, . ., 3), H_{1}=t_{1}+\frac{h_{1}+h_{2}}{2}, H_{2}=t_{2}+\frac{h_{2}+h_{3}}{2} .
$$
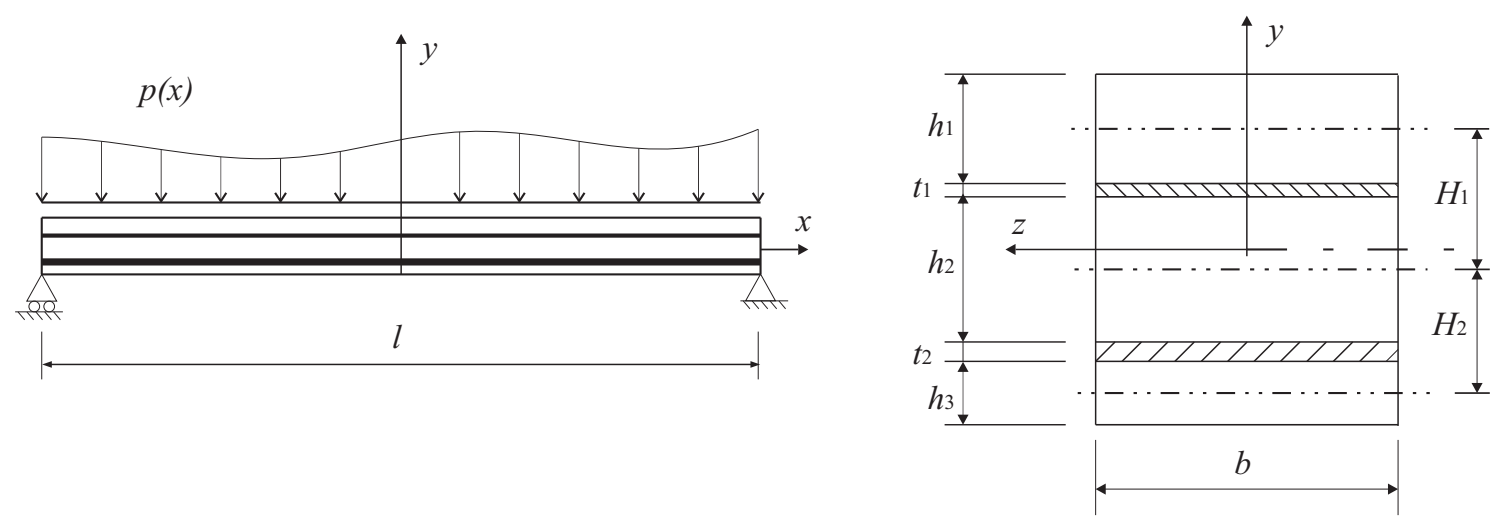

Figure 1: Five-layered simply-supported laminated glass beam composed of three glass plies bonded by polymeric interlayers. Longitudinal view and cross section (not in the same scale).

\section{$2.1 \quad$ The model}

Under the hypotheses that glass-polymer bond is perfect and the interlayer strain in direction $y$ is negligible, provided that strains are small and rotations moderate, the kinematics is completely described by the vertical displacement $v(x)$, the same for the three glass components, and the horizontal displacements $u_{1}(x), u_{2}(x)$ and $u_{3}(x)$ of the centroid of the cross-sectional areas of glass plies. Following the same procedure of [13], the shear strain in the two interlayers is given by

$$
\gamma_{1}(x)=\frac{1}{t_{1}}\left[u_{1}(x)-u_{2}(x)+v^{\prime}(x) H_{1}\right], \quad \gamma_{2}(x)=\frac{1}{t_{2}}\left[u_{2}(x)-u_{3}(x)+v^{\prime}(x) H_{2}\right] .
$$


The total strain energy of the laminated glass beam [15] is provided by the flexural and extensional contributions of the three glass layers, by the part corresponding to the shear deformation of the interlayers, by the work of the external loads $p(x)$, and reads

$$
\begin{aligned}
& \mathfrak{E}\left[u_{1}(x), u_{2}(x), u_{3}(x), v(x)\right]= \\
& \quad \int_{-l / 2}^{l / 2}\left\{\frac { 1 } { 2 } \left[E\left(I_{1}+I_{2}+I_{3}\right) v^{\prime \prime}(x)^{2}+E\left[A_{1} u_{1}^{\prime}(x)^{2}+A_{2} u_{2}^{\prime}(x)^{2}+A_{3} u_{3}^{\prime}(x)^{2}\right]+\right.\right. \\
& \left.\left.+G b\left[\frac{1}{t_{1}}\left(u_{1}(x)-u_{2}(x)+v^{\prime}(x) H_{1}\right)^{2}+\frac{1}{t_{1}}\left(u_{2}(x)-u_{3}(x)+v^{\prime}(x) H_{2}\right)^{2}\right]\right]+p(x) v(x)\right\} d x .
\end{aligned}
$$

The first variation of this functional with respect to the variables $v(x)$ and $u_{i}(x), i=1, . ., 3$, gives respectively the Euler-Lagrange equilibrium equations

$E\left(I_{1}+I_{2}+I_{3}\right) v^{\prime \prime \prime \prime \prime}(x)-G b\left[\frac{H_{1}}{t_{1}}\left(u_{1}(x)-u_{2}(x)+v^{\prime}(x) H_{1}\right)^{\prime}+\frac{H_{2}}{t_{2}}\left(u_{2}(x)-u_{3}(x)+v^{\prime}(x) H_{2}\right)^{\prime}\right]+p(x)=0$,

$$
E A_{1} u_{1}^{\prime \prime}(x)-\frac{G b}{t_{1}}\left(u_{1}(x)-u_{2}(x)+v^{\prime}(x) H_{1}\right)=0
$$

$$
\begin{gathered}
E A_{2} u_{2}^{\prime \prime}(x)-G b\left[-\frac{1}{t_{1}}\left(u_{1}(x)-u_{2}(x)+v^{\prime}(x) H_{1}\right)+\frac{1}{t_{2}}\left(u_{2}(x)-u_{3}(x)+v^{\prime}(x) H_{2}\right)\right]=0, \\
E A_{3} u_{3}^{\prime \prime}(x)+\frac{G b}{t_{2}}\left(u_{2}(x)-u_{3}(x)+v^{\prime}(x) H_{2}\right)=0 .
\end{gathered}
$$


Recalling that $E A_{i} u_{i}^{\prime \prime}(x)$ is the derivative of the axial force $N_{i}(x)$ in the $i-t h$ glass layer, conditions (2.5), (2.6) and (2.7) represent the axial equilibrium of the glass plies under the mutual shear force per unit length $\tau_{i}(x)=G b \gamma_{i}(x)$, transmitted by the polymeric interlayer, as schematically represented in Fig. 2.a. Moreover, from fig. 2.b, it can be observed that the shear coupling offered by the upper interlayer is statically equipollent to a distributed torque per unit length in the upper glass ply (layer 1 ) equal to $-\left(h_{1} / 2+t_{1}^{*}\right) \tau_{1}(x)$, where $t_{1}^{*}$ is arbitrary, and equal to $-\left(h_{2} / 2+t_{1}-t_{1}^{*}\right) \tau_{1}(x)$ in the middle ply (layer 2$)$. Similarly, the shear stress in the lower interlayer gives in the middle glass ply (layer 2) the distributed torque per unit length $-\left(h_{2} / 2+t_{2}^{*}\right) \tau_{2}(x)$, with $t_{2}^{*}$ arbitrary, and $-\left(h_{3} / 2+t_{2}-t_{2}^{*}\right) \tau_{2}(x)$ in the bottom ply (layer 3). Summing up the contributions, the total torque per unit length acting on the whole layered beam is equal to

$$
m(x)=-\left(\frac{h_{1}+h_{2}}{2}+t_{1}\right) \tau_{1}(x)-\left(\frac{h_{2}+h_{3}}{2}+t_{2}\right) \tau_{2}(x)=-H_{1} \tau_{1}(x)-H_{2} \tau_{2}(x) .
$$

Hence, (2.4) represents the equilibrium equation of the whole laminated package under bending, due to the external load $p(x)$ and a distributed torque per unit length $m(x)$.

In conclusion, equations (2.4), (2.5), (2.6) and (2.7) may be conveniently rewritten as

$$
\begin{aligned}
& E I v^{\prime \prime \prime \prime}(x)+p(x)+m^{\prime}(x)=0, \\
& N_{1}^{\prime}(x)=\tau_{1}(x) \\
& N_{2}^{\prime}(x)=-\tau_{1}(x)+\tau_{2}(x), \\
& N_{3}^{\prime}(x)=-\tau_{2}(x)
\end{aligned}
$$

Standard arguments in the calculus of variation [16] furnish the boundary conditions 

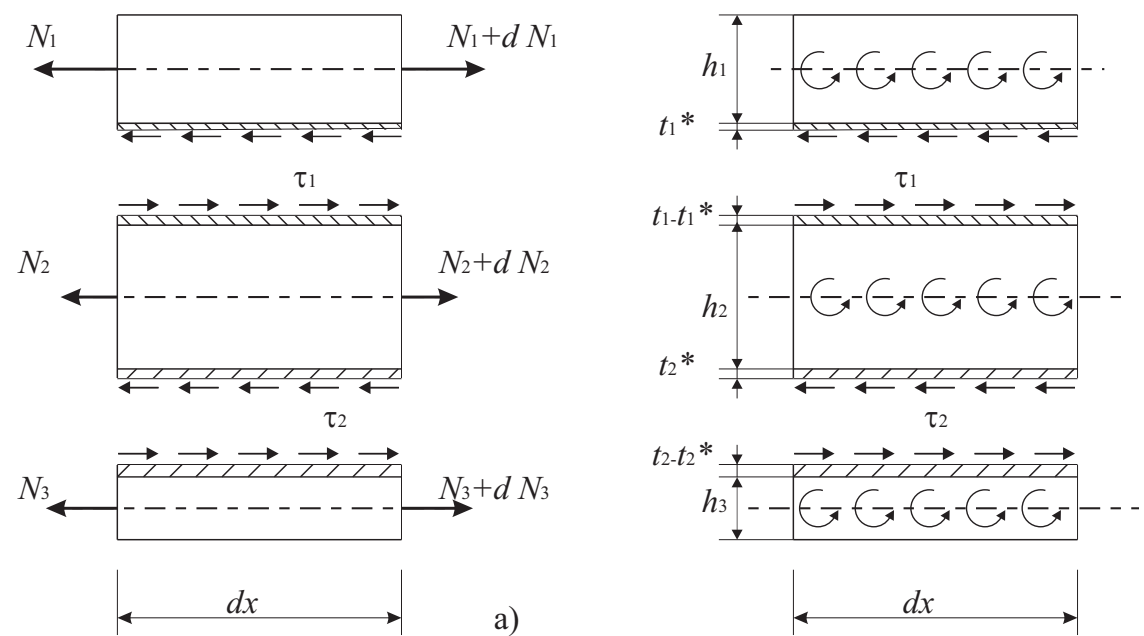

b)

Figure 2: Physical interpretation of Euler-Lagrange equations.

$$
\begin{aligned}
& {\left[-\left(E\left(I_{1}+I_{2}+I_{3}\right) v^{\prime \prime \prime}(x)+G b\left(\gamma_{1} H_{1}+\gamma_{2} H_{2}\right)\right) \delta v(x)\right]_{-l / 2}^{l / 2}=0} \\
& {\left[E\left(I_{1}+I_{2}+I_{3}\right) v^{\prime \prime}(x) \delta v^{\prime}(x)\right]_{-l / 2}^{l / 2}=0} \\
& {\left[E A_{1} u_{1}^{\prime}(x) \delta u_{1}(x)\right]_{-l / 2}^{l / 2}=0} \\
& {\left[E A_{2} u_{2}^{\prime}(x) \delta u_{2}(x)\right]_{-l / 2}^{l / 2}=0} \\
& {\left[E A_{3} u_{3}^{\prime}(x) \delta u_{2}(x)\right]_{-l / 2}^{l / 2}=0}
\end{aligned}
$$

where $\delta v(x), \delta u_{i}(x)(i=1, . ., 3)$ denote the variations of $v(x)$ and $u_{i}(x)$, respectively. The meaning of (2.10) is that, in order to rended each term equal to zero, at the boundary points $x= \pm l / 2$ one can either prescribe the value of the unknown field, so that the corresponding variation is null (geometric boundary condition); or when the variation is arbitrary because the value at the boundary is not prescribed, the corresponding coefficient must be zero (natural boundary condition). For example, if the $i-t h$ glass layer the beam is not constrained at, say, $x=l / 2$, so that $\delta u_{i}(l / 2) \neq 0$, then the correspondent axial force $E A_{i} u_{i}^{\prime}(l / 2)$ must be null.

A relationship between the horizontal displacement of the glass plies can be found by 
rearranging equations (2.5), (2.6) and (2.7) in the form

$$
\begin{aligned}
& \left(E A_{1} u_{1}^{\prime}(x)+E A_{2} u_{2}^{\prime}(x)+E A_{3} u_{3}^{\prime}(x)\right)^{\prime}=0 \\
& \Rightarrow E A_{1} u_{1}^{\prime}(x)+E A_{2} u_{2}^{\prime}(x)+E A_{3} u_{3}^{\prime}(x)=N_{1}(x)+N_{2}(x)+N_{3}(x)=\text { const }=: N,
\end{aligned}
$$

where $N$ represents the constant resultant axial force. This finding is not surprising because no axially oriented force is supposed to act on the beam.

If the axial displacement of the glass plies is not constrained at one of extremities, say $x=-l / 2$, so that $\delta u_{i}(-l / 2) \neq 0$ and from $(2.10)_{3-4-5} E A_{i} u_{i}^{\prime}(-l / 2)=0(i=1, . ., 3)$, equation (2.11) leads to $N_{1}(x)+N_{2}(x)+N_{3}(x)=0, \forall x \in(-l / 2, l / 2)$. In the most general case in which the beam is constrained at both its ends so that $\delta u_{i}( \pm l / 2)=0$, we suppose that no overall axial elongation is given to each glass ply, i.e., $\int_{-l / 2}^{l / 2} u_{i}^{\prime}(x) d x=0(i=1, . ., 3)$. This allows to evaluate from (2.11)

$$
0=\int_{-l / 2}^{l / 2}\left(E A_{1} u_{1}^{\prime}(x)+E A_{2} u_{2}^{\prime}(x)+E A_{3} u_{3}^{\prime}(x)\right) d x=\int_{-l / 2}^{l / 2} N d x=N l \Rightarrow N=0 .
$$

In conclusion, in both cases the following relation holds:

$$
\begin{aligned}
& N_{1}(x)+N_{2}(x)+N_{3}(x)=\left(E A_{1} u_{1}(x)+E A_{2} u_{2}(x)+E A_{3} u_{3}(x)\right)^{\prime}=0 \\
& \Rightarrow \quad A_{1} u_{1}(x)+A_{2} u_{2}(x)+A_{3} u_{3}(x)=\text { const. }
\end{aligned}
$$

In order to prevent the rigid body motion in $x$ direction, the horizontal displacement has to be constrained. Since this constrain is quite arbitrary, we suppose that it is such that 


$$
A_{1} u_{1}(x)+A_{2} u_{2}(x)+A_{3} u_{3}(x)=0 .
$$

In other words, there is always a horizontal rigid translation for which the constant in (2.13) is null. Condition (2.14) allows noteworthy simplifications in the following analysis.

\subsection{Layered and monolithic limits}

It should be observed that whenever $G \rightarrow 0$, the Euler's equations (2.4), (2.5), (2.6) and (2.7) become

$$
\left\{\begin{array}{l}
E\left(I_{1}+I_{2}+I_{3}\right) v^{\prime \prime \prime \prime}(x)+p(x)=0, \\
E A_{1} u_{1}^{\prime \prime}(x)=0, \\
E A_{2} u_{2}^{\prime \prime}(x)=0, \\
E A_{3} u_{3}^{\prime \prime}(x)=0 .
\end{array}\right.
$$

It can be recognized that these correspond to the the equilibrium of three frictionless sliding glass beams. This case is the layered limit.

In the case that $G \rightarrow+\infty$, a relationship between horizontal and vertical displacements can be obtained by imposing that $\gamma_{1}(x)=0$ and $\gamma_{2}(x)=0$. Using (2.2) and adding condition (2.14), a system of equations is obtained in the form

$$
\left\{\begin{array} { l } 
{ h _ { 1 } u _ { 1 } ( x ) + h _ { 2 } u _ { 2 } ( x ) + h _ { 3 } u _ { 3 } ( x ) = 0 , } \\
{ u _ { 1 } ( x ) - u _ { 2 } ( x ) + v ^ { \prime } ( x ) H _ { 1 } = 0 , } \\
{ u _ { 2 } ( x ) - u _ { 3 } ( x ) + v ^ { \prime } ( x ) H _ { 2 } = 0 , }
\end{array} \Rightarrow \left\{\begin{array}{l}
u_{1}(x)=-\frac{h_{2} H_{1}+h_{3}\left(H_{1}+H_{2}\right)}{h_{1}+h_{2}+h_{3}} v^{\prime}(x) \\
u_{2}(x)=\frac{h_{1} H_{1}-h_{3} H_{2}}{h_{1}+h_{2}+h_{3}} v^{\prime}(x) \\
u_{3}(x)=\frac{h_{2} H_{2}+h_{1}\left(H_{1}+H_{2}\right)}{h_{1}+h_{2}+h_{3}} v^{\prime}(x)
\end{array}\right.\right.
$$


Denoting with $d_{i}(i=1, . ., 3)$ the distance with sign (positive in the direction of increasing $y$ ) of the mid-plane of the $i$-th glass layer from the mid-plane of the whole laminated glass beam (shown in figure 1), it can be easily found that

$$
\begin{aligned}
& d_{1}=\frac{h_{2} H_{1}+h_{3}\left(H_{1}+H_{2}\right)}{h_{1}+h_{2}+h_{3}}, \\
& d_{2}=-\frac{h_{1} H_{1}-h_{3} H_{2}}{h_{1}+h_{2}+h_{3}}, \\
& d_{3}=-\frac{h_{2} H_{2}+h_{1}\left(H_{1}+H_{2}\right)}{h_{1}+h_{2}+h_{3}} .
\end{aligned}
$$

Hence, relations (2.16) may be rearranged to give

$$
u_{i}(x)=-d_{i} v^{\prime}(x), \quad i=1, . .3
$$

Then, using the first and fourth equation of (2.9), the shear force per unit length transmitted by the interlayer can be simply written as

$$
\left\{\begin{array}{l}
b \tau_{1}(x)=E A_{1} u_{1}^{\prime \prime}(x)=-E A_{1} d_{1} v^{\prime \prime \prime}(x) \\
b \tau_{2}(x)=-E A_{3} u_{3}^{\prime \prime}(x)=E A_{3} d_{3} v^{\prime \prime \prime}(x)
\end{array}\right.
$$

while in the first relationship of $(2.9)$ the term $m^{\prime}(x)$ can be rewritten as

$m^{\prime}(x)=-b\left[\tau_{1}(x) H_{1}+\tau_{2}(x) H_{2}\right]^{\prime}=E\left(A_{1} d_{1} H_{1}-A_{3} d_{3} H_{3}\right) v^{\prime \prime \prime \prime}(x)=E\left(A_{1} d_{1}^{2}+A_{2} d_{2}^{2}+A_{3} d_{3}^{2}\right) v^{\prime \prime \prime \prime}(x)$.

In conclusion, the relevant equations of the system (2.9) reduce to 


$$
\left\{\begin{array}{l}
E I_{t o t} v^{\prime \prime \prime \prime}(x)+p(x)=0, \\
b \tau_{1}(x)=-E A_{1} d_{1} v^{\prime \prime \prime}(x), \\
b \tau_{2}(x)=E A_{3} d_{3} v^{\prime \prime \prime}(x),
\end{array}\right.
$$

where $I_{t o t}$ is the moment of inertia associated with the monolithic limit, given by

$$
I_{\text {tot }}=I_{1}+I_{2}+I_{3}+A_{1} d_{1}^{2}+A_{2} d_{2}^{2}+A_{3} d_{3}^{2}
$$

This expression is quite important because, in general, the monolithic limit is erroneously associated with a ply whose thickness is the sum of the thicknesses of the glass plies; on the other hand, (2.22) gives the inertia of the glass layers properly spaced of the interlayer gaps $t_{1}$ and $t_{2}$ (Figure 1). The last two equations in (2.21) are the shear stress in the interlayers, calculated according with Jourawski's formulation.

\subsection{The Enhanced Effective Thickness (EET) approach}

Once external load $p(x)$ and boundary conditions are given, the fields $v(x)$ and $u_{i}(x)$ can be determined by integrating the differential system (2.4), (2.5), (2.6) and (2.7), with boundary conditions derived from (2.10). An approximation can be found by choosing an appropriate class of shape functions for the unknown fields $v(x)$ and $u_{i}(x)$, defined up to a few parameters that will be determined from energy minimization. This procedure has already been used in [13] for the case of three-layered beams.

The shape functions for the vertical displacement $v(x)$ and for the horizontal displacements $u_{i}(x)(i=1, \ldots, 3)$, must be compatible with the qualitative properties of the exact solution and, in particular, must comprehend the monolithic-limit solution, when $G \rightarrow \infty$, 
and the layered-limit solution, when $G \rightarrow 0$. In terms of the field $v(x)$, such borderline cases correspond, respectively, to the fields $v_{M}(x)$ and $v_{L}(x)$ that, being the solutions of the differential equations

$$
E I_{t o t} v_{M}^{\prime \prime \prime \prime}(x)+p(x)=0, \quad E\left(I_{1}+I_{2}+I_{3}\right) v_{L}^{\prime \prime \prime \prime}(x)+p(x)=0,
$$

have to be of the form

$$
v_{M}(x)=-\frac{g(x)}{E I_{\text {tot }}}, \quad v_{L}(x)=-\frac{g(x)}{E\left(I_{1}+I_{2}+I_{3}\right)},
$$

where $g(x)$ is a function that is uniquely determined from the form of the external load and the geometric boundary conditions of the beam. It is thus natural to think of an approximating class of solutions in the form

$$
v(x)=-\frac{g(x)}{E I_{R}}
$$

where $g(x)$ plays the role of a shape function for the vertical displacement and $I_{R}$ is the effective moment of inertia of the laminate glass beam, comprised between the values $\left(I_{1}+\right.$ $I_{2}+I_{3}$ ), corresponding to the layered limit, and $I_{t o t}$, associated with the monolithic limit. It is convenient to define $I_{R}$ as the weighted harmonic mean of this two values through the parameter $\eta$, a non-dimensional quantity tuning the plate response from the layered limit $(\eta=0)$ to the monolithic limit $(\eta=1)$. In conclusion, we can write

$$
\frac{1}{I_{R}}=\frac{\eta}{I_{t o t}}+\frac{1-\eta}{I_{1}+I_{2}+I_{3}}
$$


For what the horizontal displacement is concerned, consider first the layered and monolithic limit. In the layered limit, one finds from the last three equations of (2.15) that the horizontal displacement, say $u_{i L}(x)(i=1, . ., 3)$ to distinguish, is a linear function. But if we suppose, as done to derive (2.12), that the overall axial elongation in each glass ply is zero, it can be easily concluded that $u_{i L}=0(i=1, . ., 3)$, which is also compatible with condition (2.14). In the monolithic limit, the horizontal displacements, say $u_{i M}(x)(i=1, . ., 3)$, are related with the vertical component $v(x)$ through equations (2.18). In conclusion, one has

$$
u_{i L}=0, u_{i M}=-d_{i} v^{\prime}(x)=d_{i} \frac{g^{\prime}(x)}{E I_{t o t}}, \quad i=1, . ., 3
$$

so that an approximate solution for the horizontal displacement can be sought in the form

$$
u_{i}(x)=\beta d_{i} \frac{g^{\prime}(x)}{E I_{t o t}}
$$

where $\beta$ is another nondimensional parameter, again tuning the response from the layered limit $(\beta=0)$, implying null horizontal force in the glass layers, to the monolithic limit $(\beta=1)$, leading to $\gamma_{i}(x)=0, i=1,2$.

The corresponding strain energy (2.3) can thus be re-written as a function of the shape function and the parameters $\eta$ and $\beta$ in the form 


$$
\begin{aligned}
& \mathfrak{E}\left[u_{1}(x), u_{2}(x), u_{3}(x), v(x)\right]=\widehat{\mathfrak{E}}[\eta, \beta, g(x)] \\
& =\int_{-l / 2}^{l / 2}\left\{\frac{\left(I_{1}+I_{2}+I_{3}\right)}{2 E}\left[\frac{\eta}{I_{t o t}}+\frac{1-\eta}{I_{1}+I_{2}+I_{3}}\right]^{2}\left[g^{\prime \prime}(x)\right]^{2}+\frac{\beta^{2}}{2 E I_{\text {tot }}^{2}}\left(A_{1} d_{1}^{2}+A_{2} d_{2}^{2}+A_{3} d_{3}^{2}\right)\left[g^{\prime \prime}(x)\right]^{2}\right. \\
& +\frac{G b}{2 E^{2} I_{t o t}}\left[\frac{\left(\frac{\beta\left(-d_{1}+d_{2}\right)}{I_{\text {tot }}}-\left(\frac{\eta}{I_{\text {tot }}}+\frac{1-\eta}{t_{1}+I_{2}+I_{3}}\right) H_{1}\right)^{2}}{t_{1}}+\frac{\left(\frac{\beta\left(-d_{2}+d_{3}\right)}{I_{\text {tot }}}-\left(\frac{\eta}{I_{\text {tot }}}+\frac{1-\eta}{t_{2}+I_{2}+I_{3}}\right) H_{2}\right)^{2}}{t_{2}}\right]\left[g^{\prime}(x)\right]^{2} \\
& \left.-\frac{p(x)}{E}\left[\frac{\eta}{I_{t o t}}+\frac{1-\eta}{I_{1}+I_{2}+I_{3}}\right] g(x)\right\} d x,
\end{aligned}
$$

in which $g(x)$ is given because its depends on loading and boundary conditions for the beam. Moreover, noticing that $d_{1}-d_{2}=H_{1}$ and $d_{2}-d_{3}=H_{2}$ (figure 1 ), the strain energy may be simplified as

$$
\begin{aligned}
& \mathfrak{E}\left[u_{1}(x), u_{2}(x), u_{3}(x), v(x)\right]=\widehat{\mathfrak{E}}[\eta, \beta, g(x)] \\
= & \int_{-l / 2}^{l / 2}\left\{\frac{\left(I_{1}+I_{2}+I_{3}\right)}{2 E}\left[\frac{\eta}{I_{t o t}}+\frac{1-\eta}{I_{1}+I_{2}+I_{3}}\right]^{2}\left[g^{\prime \prime}(x)\right]^{2}+\frac{\beta^{2}}{2 E I_{\text {tot }}^{2}}\left(A_{1} d_{1}^{2}+A_{2} d_{2}^{2}+A_{3} d_{3}^{2}\right)\left[g^{\prime \prime}(x)\right]^{2}\right. \\
& \left.+\frac{G b}{2 E^{2} I_{t o t}}\left(\frac{H_{1}^{2}}{t_{1}}+\frac{H_{2}^{2}}{t_{2}}\right)\left[\frac{\beta}{I_{t o t}}+\left(\frac{\eta}{I_{t o t}}+\frac{1-\eta}{I_{1}+I_{2}+I_{3}}\right)\right]^{2}\left[g^{\prime}(x)\right]^{2}-\frac{p(x)}{E}\left[\frac{\eta}{I_{t o t}}+\frac{1-\eta}{I_{1}+I_{2}+I_{3}}\right] g(x)\right\} d x .
\end{aligned}
$$

Minimization has to be performed with respect to the free parameters $\eta$ and $\beta$ only. Conditions $\frac{\partial \widehat{\mathbb{E}}}{\partial \beta}=0$ and $\frac{\partial \widehat{\mathfrak{E}}}{\partial \eta}=0$ lead respectively to 


$$
\begin{aligned}
& \left(I_{1}+I_{2}+I_{3}\right)\left[\frac{\eta}{I_{t o t}}+\frac{1-\eta}{I_{1}+I_{2}}\right] \int_{-l / 2}^{l / 2}\left[g^{\prime \prime}(x)\right]^{2} d x \\
& -\frac{G b}{E}\left(\frac{H_{1}^{2}}{t_{1}}+\frac{H_{2}^{2}}{t_{2}}\right)\left[\frac{\beta}{I_{t o t}}-\frac{\eta}{I_{t o t}}-\frac{1-\eta}{I_{1}+I_{2}}\right] \int_{-l / 2}^{l / 2}\left[g^{\prime}(x)\right]^{2} d x-\int_{-l / 2}^{l / 2} p(x) g(x) d x=0 \\
& \frac{\beta}{I_{t o t}}\left(A_{1} d_{1}^{2}+A_{2} d_{2}^{2}+A_{3} d_{3}^{2}\right) \int_{-l / 2}^{l / 2}\left[g^{\prime \prime}(x)\right]^{2} d x \\
& +\frac{G b}{E}\left(\frac{H_{1}^{2}}{t_{1}}+\frac{H_{2}^{2}}{t_{2}}\right)\left[\frac{\beta}{I_{t o t}}-\frac{\eta}{I_{t o t}}-\frac{1-\eta}{I_{1}+I_{2}}\right] \int_{-l / 2}^{l / 2}\left[g^{\prime}(x)\right]^{2} d x=0
\end{aligned}
$$

As done in [13], these expressions may be simplified by observing from (2.23) and (2.24) that $v(x)$ of $(2.25)$ is the exact solution of the elastic bending of a beam with constant flexural inertia $I_{R}$ under the same load $p(x)$ and boundary condition of the problem. Consider the virtual work equality for this system in which the aforementioned $v(x)$ is selected as the strain/displacement field, whereas the bending moment in equilibrium with $p(x)$ is given by $M(x)=v^{\prime \prime}(x) E I_{R}$. The external and internal work can be written as

$$
L_{v e}=-\frac{1}{E I_{R}} \int_{-l / 2}^{l / 2} p(x) g(x) d x, \quad L_{v i}=-\int_{-l / 2}^{l / 2} M(x) v^{\prime \prime}(x) d x=\frac{1}{E I_{R}} \int_{-l / 2}^{l / 2}\left[g^{\prime \prime}(x)\right]^{2} d x,
$$

so that one has

$$
\int_{-l / 2}^{l / 2} p(x) g(x) d x=\int_{-l / 2}^{l / 2}\left[g^{\prime \prime}(x)\right]^{2} d x
$$

This condition can be used to simplify (2.31) and (2.32), yielding the noteworthy relationship 


$$
\eta=\beta=\frac{1}{1+\frac{E}{G b I_{\text {tot }}\left(\frac{H_{1}^{2}}{t_{1}}+\frac{H_{2}^{2}}{t_{2}}\right)}\left(I_{1}+I_{2}+I_{3}\right)\left(A_{1} d_{1}^{2}+A_{2} d_{2}^{2}+A_{3} d_{3}^{2}\right) \Psi},
$$

where the coefficient $\Psi$ reads

$$
\Psi=\frac{\int_{-l / 2}^{l / 2}\left[g^{\prime \prime}(x)\right]^{2} d x}{\int_{-l / 2}^{l / 2}\left[g^{\prime}(x)\right]^{2} d x}
$$

Evidently, the value of $\Psi$ depends upon the geometry, boundary and loading conditions of the beam, and it is reported in Table 1 for the most common cases. Remarkably, these data are identical to those recorded in [17] for a three-layered beam. Indeed, (3.17) represents

\begin{tabular}{|c|c|c|c|}
\hline $\begin{array}{l}\text { Loading and boundary } \\
\text { conditions }\end{array}$ & $\Psi$ & $\begin{array}{l}\text { Loading and boundary } \\
\text { conditions }\end{array}$ & $\Psi$ \\
\hline $\begin{array}{lllll} & 1 & 1 & 1 & 1 \\
\end{array}$ & 168 & $\begin{array}{lllll} & 1 & 1 & 1 & 1 \\
\end{array}$ & 42 \\
\hline$\stackrel{n}{H} \quad l \quad l$ & $17 l^{2}$ & $l$ & $\overline{l^{2}}$ \\
\hline$\stackrel{a}{a} \rightarrow \frac{b}{l}$ & $\frac{15}{l^{2}+2 a b}$ & $\frac{1+1}{1}$ & $\frac{14}{5 l^{2}}$ \\
\hline$\underset{\leftarrow}{\stackrel{f}{r} l / 2}$ & $\frac{10}{l^{2}}$ & $\longrightarrow$ & $\frac{5}{2 l^{2}}$ \\
\hline 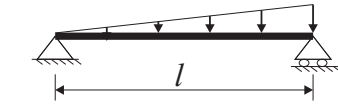 & $\frac{10}{l^{2}}$ & $\frac{1}{l}$ & $\frac{45}{14 l^{2}}$ \\
\hline 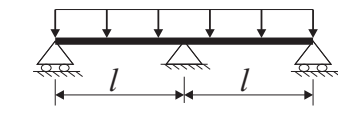 & $\frac{21}{l^{2}}$ & $\begin{array}{llll} & 1 & 1 & 1 \\
& l\end{array}$ & $\frac{21}{l^{2}}$ \\
\hline
\end{tabular}
the natural extension of the corresponding expression obtained in [13].

Table 1: Values of coefficient $\Psi$ for laminated glass beams under different boundary and load conditions.

The parameter $\eta=\beta$ can be used to define the effective thickness of the laminated glass beam. Recalling (2.26), the deflection-effective thickness $\hat{h}_{w}$, associated with the value $\eta$, can be written in the form 


$$
\frac{1}{\left(\hat{h}_{w}\right)^{3}}=\frac{\eta}{h_{1}^{3}+h_{2}^{3}+h_{3}^{3}+12\left(h_{1} d_{1}^{2}+h_{2} d_{2}^{2}+h_{3} d_{3}^{2}\right)}+\frac{(1-\eta)}{\left(h_{1}^{3}+h_{2}^{3}+h_{3}^{3}\right)} .
$$

The stress-effective thickness may be defined as the (constant) thickness $\hat{h}_{i ; \sigma}$ of a monolithic beam for which the maximum bending stress is equal to the maximum stresses in the $i-t h$ glass layer of the laminated package. The bending stress in the $i-$ th glass ply is given by

$$
\left|\sigma_{(i)}\right| \max =\frac{\max _{x}|M(x)|}{\frac{1}{6} b \hat{h}_{i ; \sigma}^{2}}=\max _{x}\left|\frac{N_{i}(x)}{A_{i}} \pm \frac{M_{i}(x)}{I_{i}} \frac{h_{i}}{2}\right|,
$$

where $N_{i}(x)=E A_{i} u_{i}^{\prime}(x)=\frac{A_{i} \beta d_{1}}{I_{\text {tot }}} g^{\prime \prime}(x)$ and $M_{i}=E I_{i} v^{\prime \prime}(x)=-\frac{I_{i}}{I_{R}} g^{\prime \prime}(x)$. By substituting such values into equation (2.38), one obtains

$$
\frac{1}{\left(\hat{h}_{i ; \sigma}\right)^{2}}=\frac{2 \eta\left|d_{i}\right|}{h_{1}^{3}+h_{2}^{3}+h_{3}^{3}+12\left(h_{1} d_{1}^{2}+h_{2} d_{2}^{2}+h_{3} d_{3}^{2}\right)}+\frac{h_{i}}{\hat{h}_{w}^{3}}
$$

The maximal stress in the $i$-th glass ply is calculated by considering a monolithic beam of thickness $\hat{h}_{i ; \sigma}$ under the same loading and boundary condition of the layered beam.

\section{Multi-layered beams with glass plies of equal thickness}

Consider now a package composed by $N$ glass plies of equal thickness $h$, bonded by $N-1$ polymeric interlayers of thickness $t$ (Figure 3). Again, the beam length is $l$, its width is $b$, while $E$ denotes the Young's modulus of glass and $G$ is the shear modulus of the polymer.

\subsection{The strain energy functional}

Let 


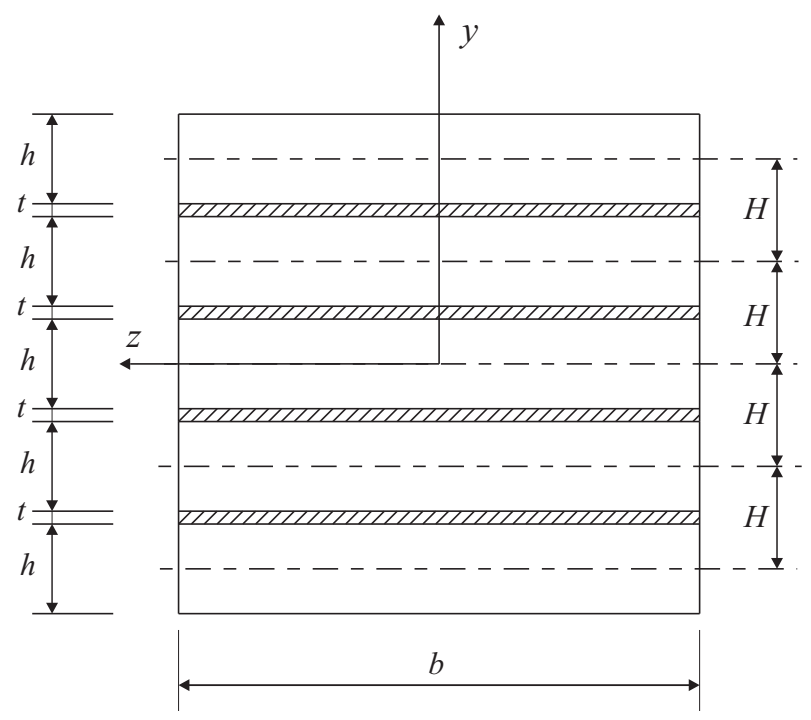

Figure 3: Laminated glass package composed of $N(N=5$ in the picture) glass plies bonded by polymeric interlayers.

$$
A=h b ; I=\frac{b h^{3}}{12} ; H=t+h
$$

Under the same hypotheses of section 2 , and denoting with $u_{i}(x)$ the horizontal displacement of the centroid of the $i$-th glass ply, the shear strain in the $i$-th interlayer may be written as $\gamma_{i}(x)=\frac{1}{t}\left[u_{i}(x)-u_{i+1}(x)+v^{\prime}(x) H\right](i=1, . ., N-1)$. Analogously with equation (2.3), the strain energy of the laminated glass beam is

$$
\begin{aligned}
\mathfrak{E}\left[u_{i}(x), v(x)\right] & =\int_{-l / 2}^{l / 2}\left\{\frac{1}{2}\left[E N I v^{\prime \prime}(x)^{2}+E A \sum_{i=1}^{N} u_{i}^{\prime}(x)^{2}\right]+\right. \\
& \left.+\frac{G b}{t} \sum_{i=1}^{N-1}\left[u_{i}(x)-u_{i+1}(x)+v^{\prime}(x) H\right]^{2}+p(x) v(x)\right\} d x .
\end{aligned}
$$

Minimization of this functional with respect to $v(x)$ and $u_{i}(x),(i=1, . ., N)$ leads to the $N+1$ Euler-Lagrange equations 


$$
E N I v^{\prime \prime \prime \prime}(x)-\frac{G b H}{t} \sum_{i=1}^{N-1}\left[u_{i}(x)-u_{i+1}(x)+v^{\prime}(x) H\right]^{\prime}+p(x)=0,
$$

$$
\begin{aligned}
& E A u_{1}^{\prime \prime}(x)-\frac{G b}{t}\left(u_{1}(x)-u_{2}(x)+v^{\prime}(x) H\right)=0, \\
& E A u_{i}^{\prime \prime}(x)+\frac{G b}{t}\left[\left(u_{i-1}(x)-u_{i}(x)+v^{\prime}(x) H\right)-\left(u_{i}(x)-u_{i+1}(x)+v^{\prime}(x) H\right)\right]=0, i=2, . ., N-1, \\
& E A u_{N}^{\prime \prime}(x)+\frac{G b}{t}\left(u_{N-1}(x)-u_{N}(x)+v^{\prime}(x) H\right)=0,
\end{aligned}
$$

with boundary conditions

$$
\begin{aligned}
& {\left[-\left(E N I v^{\prime \prime \prime}(x)+\frac{G b H}{t} \sum_{i=1}^{N-1}\left[u_{i}(x)-u_{i+1}(x)+v^{\prime}(x) H\right]\right) \delta v(x)\right]_{-l / 2}^{l / 2}=0} \\
& {\left[E N I v^{\prime \prime}(x) \delta v^{\prime}(x)\right]_{-l / 2}^{l / 2}=0} \\
& {\left[E A u_{i}^{\prime}(x) \delta u_{i}(x)\right]_{-l / 2}^{l / 2}=0 \quad \forall i=1, . ., N}
\end{aligned}
$$

where $\delta v(x), \delta u_{i}(x)(i=1, . ., N)$ denote the variations of $v(x)$ and $u_{i}(x)$. The interpretation of these equations is the same of Section 2.

Furthermore, with the same argument that has led to (2.13), it can be demonstrate that a relationship between horizontal displacements of the glass plies exists, of the type

$$
\sum_{i=1}^{N} u_{i}(x)=0
$$

which is of this simple form because all the glass plies have the same cross sectional area. If the glass plies were of different thickness, in (3.6) each term $u_{i}(x)$ would have been multiplied by the corresponding cross sectional area $A_{i}$ as in (2.14), but it would be difficult then to 
find simple formulas for the most general case.

\subsection{Layered and monolithic limit}

Whenever the layered limit is attain, the governing equilibrium equations of the laminated glass beam can be obtained simply by imposing $G=0$ in (3.2) so that

$$
\left\{\begin{array}{l}
E N I v^{\prime \prime \prime \prime}(x)+p(x)=0, \\
E A u_{i}^{\prime \prime}(x)=0 . \forall i=1, . ., N
\end{array}\right.
$$

On the other hand, whenever $G \rightarrow \infty$, the shear strain in the interlayer tends to zero, i.e, $\gamma_{i}(x)=0, i=1, . ., N-1$, leading to

$$
u_{i+1}(x)=u_{i}(x)+v^{\prime}(x) H, \quad \forall i=1, . ., N-1 .
$$

Henceforth, relationships between horizontal and vertical displacements may be obtain by solving the system of equations (3.8) and (3.6). One obtains

$$
u_{1}=\frac{2 i-(N+1)}{2} v^{\prime}(x) H=-d_{i} v^{\prime}(x) H
$$

where $d_{i}=\frac{(N+1)-2 i}{2} H$ is again the distance with sign of the mid-plane of the $i-$ th glass ply from the mid-plane of the laminated glass package, positive with increasing $y$ (figure 3 ). The shear force per unit length $\tau_{i}(x)=G b \gamma_{i}(x)$ can be evaluated by rearranging equations (3.4), to give 


$$
\tau_{i}(x)=G b \gamma_{i}(x)=E A \sum_{j=1}^{i} u_{j}^{\prime \prime}(x)=E A v^{\prime \prime \prime}(x) H \sum_{j=1}^{i} \frac{2 i-(N+1)}{2}=E A v^{\prime \prime \prime}(x) H \frac{i(i-N)}{2}
$$

Now, the second term of (3.3) can be evaluated as

$$
G b H \sum_{i=1}^{N-1} \gamma_{i}^{\prime}(x)=E A H^{2} v^{\prime \prime \prime \prime}(x) \sum_{i=1}^{N-1} \frac{i(i-N)}{2}=-\frac{E A H^{2}}{12}(N-1) N(N+1) v^{\prime \prime \prime \prime}(x) .
$$

By substituting such result in (3.3), the equilibrium equations for the monolithic limit turn out to be

$$
E I_{\text {tot }} v^{\prime \prime \prime \prime}(x)+p(x)=0,
$$

where $I_{t o t}=N I+\frac{A H^{2}}{12}(N-1) N(N+1)$ represents the moment of inertia of a monolithic plate, whose inertia is that of the two glass plies properly spaced of the gap given by the thickness of the interlayer. It can be shown that this quantity corresponds to

$$
I_{\text {tot }}=N I+A \sum_{i=1}^{N} d_{i}^{2}=N I+A \sum_{i=1}^{N}\left(\frac{(N+1)-2 i}{2} H\right)^{2}=N I+\frac{A H^{2}}{12}(N-1) N(N+1),
$$

which represents the analogous of (2.22). 


\subsection{The EET approach}

Following the same procedure of Section 2.3, the solution for the vertical displacement can be found in the form (2.25), where $g(x)$ is a shape function, chosen according to the loading and boundary condition, and the effective moment of inertia of the beam is defined as

$$
\frac{1}{I_{R}}=\frac{\eta}{I_{t o t}}+\frac{1-\eta}{N \cdot I}
$$

The shape function for the horizontal displacement can be found of the same form (2.28). Under these assumption, since $d_{i}-d_{i+1}=H$, the shear strain in the $i$ th interlayer is

$$
\gamma_{i}=\frac{H}{E t}\left[\frac{\beta}{I_{t o t}}-\left(\frac{\eta}{I_{t o t}}+\frac{1-\eta}{N I}\right)\right] g^{\prime}(x), \quad i=1, . ., N-1
$$

Henceforth, the strain energy (3.2) may be written as

$$
\begin{aligned}
& \mathfrak{E}\left[u_{i}(x), v(x)\right]=\widehat{\mathfrak{E}}[\eta, \beta, g(x)]= \\
= & \int_{-l / 2}^{l / 2}\left\{\frac{N I}{2 E}\left[\frac{\eta}{I_{t o t}}+\frac{1-\eta}{N I}\right]^{2}\left[g^{\prime \prime}(x)\right]^{2}+\frac{\beta^{2}}{2 E I_{t o t}^{2}} \frac{A H^{2}(N-1) N(N+1)}{12}\left[g^{\prime \prime}(x)\right]^{2}+\right. \\
+ & \left.\frac{G b H^{2}(N-1)}{2 t E^{2}}\left[\frac{\beta}{I_{t o t}}-\left(\frac{\eta}{I_{t o t}}+\frac{1-\eta}{N I}\right)\right]^{2} g\left[g^{\prime}(x)\right]^{2}-\frac{p(x)}{E}\left[\frac{\eta}{I_{t o t}}+\frac{1-\eta}{N I}\right] g(x)\right\} d x,
\end{aligned}
$$

where, we recall, $g(x)$ is known.

Analogously to Section 2.3, the minimization of such energy with respect to the parameter $\eta$ and $\beta$ leads to 


$$
\eta=\beta=\frac{1}{1+\frac{E t}{G b} \frac{N^{2} I A(N+1)}{I_{t o t}} \Psi},
$$

where the coefficient $\Psi$ is again given by (2.36) and recorded in Table 1 for several cases of relevant importance. Finally, in analogy with equations (2.37) and (2.39), it can be shown that the deflection- and stress-effective thickness can be defined as

$$
\begin{gathered}
\frac{1}{\left(\hat{h}_{w}\right)^{3}}=\frac{\eta}{N h^{3}+h H^{2}(N-1) N(N+1)}+\frac{(1-\eta)}{N h^{3}}, \\
\frac{1}{\left(\hat{h}_{i ; \sigma}\right)^{2}}=\frac{2 \eta\left|d_{i}\right|}{N h^{3}+h H^{2}(N-1) N(N+1)}+\frac{h}{\hat{h}_{w}^{3}} .
\end{gathered}
$$

It is evident that the maximum stress occurs in the external glass plies, i.e. for $i=1$ and $i=N$. Since the absolute value of the distance of the mid-plane from the mid-plane of the laminated package is $\frac{N+1}{2} H$, the maximum bending stress in the laminated package may be calculated by using the stress-effective thickness of the external plies

$$
\frac{1}{\left(\hat{h}_{\sigma E X T}\right)^{2}}=\frac{\eta(N+1) H}{N h^{3}+h H^{2}(N-1) N(N+1)}+\frac{h}{\hat{h}_{w}^{3}} .
$$

The conclusion is that the EET approach can be easily extended to the case of multi-layered laminated beams, with no particular difficulties.

\section{Comparison with other methods}

Diverse alternative formulations have been proposed to calculate the effective thickness of laminated glass. Although having been conceived for a composite package of two glass 
layers and one interlayer, their extrapolation to the case of multi-layered laminated glass has been attempted. The aim of this Section is to compare results from these formulations with those obtainable with the EET method.

\subsection{Iterative Wölfel- Bennison method according to ASTM-E1300}

The formulation based upon the original approach by Wölfel [12], later developed by Bennison et al. $[10,18]$ and mentioned in the American standard ASTM-E1300 [9], relies upon several simplifying assumptions that, as discussed at length in [13], render it accurate for the case of simply supported beams under uniformly distributed loading.

With the same notation of (2.1), the method defines an equivalent moment of inertia $I_{e q}$ of a laminated glass beam, of length $l$ and width $b$, composed by two glass plies of thickness $h_{1}$ and $h_{2}$ bonded by one polymeric interlayer of thickness $t$, as the arithmetic mean of the inertiae of monolithic and layered limit. If $A_{i}=b h_{i}, I_{i}=b h_{i} / 12, H=\left(h_{1}+h_{2}\right) / 2+t$, then $I_{e q}$ reads

$$
I_{e q}=I_{1}+I_{2}+\Gamma \frac{A_{1} A_{2}}{A_{1}+A_{2}} H^{2}
$$

where the shear transfer coefficient $\Gamma$ is given by

$$
\Gamma=\frac{1}{1+\beta \frac{t E}{G b l^{2}} \frac{A_{1} A_{2}}{A_{1}+A_{2}}} .
$$

In particular, $\Gamma=0$ corresponds to the layered limit, whereas $\Gamma=1$ to perfect bonding. The coefficient $\beta$ depends upon load and boundary conditions and, for the most common cases, the corresponding values are recorded in [12] and [13]. Bennison et al. [10, 18] have proposed to use $\beta=9.6$ as in the case of uniformly distributed loading. 
From (4.1), it is immediate to evaluate the deflection-effective thickness

$$
h_{e f ; w}=\sqrt[3]{h_{1}^{3}+h_{2}^{3}+12 \Gamma \frac{h_{1} h_{2}}{h_{1}+h_{2}} H^{2}} .
$$

The maximum stress in glass can be estimated through the stress-effective thicknesses

$$
h_{1 ; e f ; \sigma}=\sqrt{\frac{h_{e f ; w}^{3}}{h_{1}+2 \Gamma h_{s ; 2}}}, h_{2 ; e f ; \sigma}=\sqrt{\frac{h_{e f ; w}^{3}}{h_{2}+2 \Gamma h_{s ; 1}}},
$$

where $h_{s ; i}=\frac{h_{i} H}{h_{1}+h_{2}}$.

As mentioned in [13], Wölfel-Bennison approach is accurate only for simply supported beams under uniformly distributed load and in those cases where the deflection curve is similar in type to this reference-case. Besides, it has been verified in [14] that, when applied to a two-dimensional plate, the method is reliable only when the deformed surface is cylindrical, so that the plate response is similar to that of a beam.

For the calculation of the effective thickness of multi-layered laminated glass, a rule of thumb, also suggested in an preliminary version of the project of European Standard prEN 13474 [8], indicates to iterate the procedure. In terms of deflection, one can calculate the effective thickness of the first and second glass layer $\left(h_{e f ; w ; 12}\right)$; from this, the effective thickness of the package composed by the equivalent monolith just determined and the third glass layer $\left(h_{e f ; w ; 123}\right)$, and so on.

In terms of stress, the procedure is not without ambiguity. In fact, one may calculate the stress-effective thickness of the first two glass layers $\left(h_{1 ; e f ; \sigma}, h_{2 ; e f ; \sigma}\right)$, but for the second step it is not clear whether to use $h_{1 ; e f ; \sigma}$ or $h_{2 ; e f ; \sigma}$ for the combination with the successive glass ply; in general, it should be safe to use the minor between these two values, and to maintain this choice also for the the successive steps. However, only the stress effective 
thickness of the last glass ply at the end of the iterations is significant, because all the values obtained at the previous steps are intermediate passage of no practical utility. In particular, the stress-effective thicknesses for the internal layers can never be calculated.

It should also be remarked that when the laminated glass package is composed by glass layers (or interlayers) of different thickness, the resulting values of effective thicknesses for what both stress and deflection is concerned, depend upon which layer is chosen as layer 1", i.e., upon the order in which the iterative combination is performed. Furthermore, the iterative procedure usually requires a considerable computation.

\subsection{The approach proposed by prEN-13474 and its critical discussion}

In its latest version, the project of European Standard prEN 13474 [8] suggests a very simple method, which is assumed to be valid for a package of $N$ glass layers of arbitrary thickness. With the same notation of before, the deflection- and stress-effective thicknesses may be calculated as

$$
h_{e f ; w}=\sqrt[3]{\sum_{i=1}^{N} h_{i}^{3}+12 \omega \sum_{i=1}^{N}\left(h_{i} d_{i}^{2}\right)}, \quad h_{e f ; \sigma ; i}=\sqrt{\frac{h_{e f ; w}^{3}}{h_{i}+2 \omega\left|d_{i}\right|}},
$$

where $\omega$ is a coefficient representing the degree of shear transfer, varying between 0 (layered limit) and 1 (monolithic limit). The value of $\omega$ is tabulated in [8], Table 11, as a function of $i$ ) the "interlayer stiffness family" to which the polymer belongs $\left.{ }^{1} ; i i\right)$ the environmental temperature and characteristic duration of the applied loads. It is important to note that the shear transfer coefficient $\omega$ is independent upon the geometry, the thickness of the interlayer, the loading and boundary conditions. Furthermore, no explicit formula is provided to

\footnotetext{
${ }^{1}$ The various types of polymers used as interlayers are classified in four different families according to their stiffeness.
} 
evaluate $\omega$ when the shear modulus of the interlayer, $G$, is known.

In our opinion, the formulation (4.5) should be questioned on a theoretical basis.

To illustrate, reference will be made to the case of a simply supported beam under uniformly distributed loading, composed by two glass plies only of thickness $h_{1}$ and $h_{2}$ is now considered. For this case, as proved in [13], the EET method provides a very accurate solution. The the shear transfer coefficient $\eta$ is given by (3.17) for $H_{2}, I_{3}, A_{3}=0$ and reads

$$
\eta_{2 l a y e r s}=\frac{1}{1+\frac{G b}{t E} \frac{I_{1}+I_{2}}{I_{\text {tot }}} \frac{A_{1} A_{2}}{A_{1}+A_{2}} \Psi}
$$

where the coefficient $\Psi$, depending on the loading and boundary conditions, is given by (2.36) and, for the case simply supported beam under uniform load, is $\Psi=\frac{168}{17 l^{2}}$, (for different loading and boundary conditions, values of $\Psi$ are tabulated in 1$)$. The deflectionand stress-effective thickness can be evaluated by setting $h_{3}=0$ into equations (2.37) and (2.39), respectively.

Now, in the prEN13474, the coefficient $\omega$ depends only on the stiffness family of the interlayer, hence on his shear modulus only. We can verify that such an assumption leads to a contradiction. In fact, consider two simply supported beams, one of length $l=1.5 \mathrm{~m}$ and the other of length $l=3 \mathrm{~m}$. We investigate four composite packages: i) 8.8.2 ( $h_{1}=h_{2}=8$ $\mathrm{mm}$ and $t=2 \times 0.38=0.76 \mathrm{~mm})$; ii) $8.8 .4\left(h_{1}=h_{2}=8 \mathrm{~mm}\right.$ and $\left.t=4 \times 0.38=1.52 \mathrm{~mm}\right)$; iii) $12.12 .2\left(h_{1}=h_{2}=12 \mathrm{~mm}\right.$ and $\left.t=2 \times 0.38=0.76 \mathrm{~mm}\right)$; iv) $12.12 .4\left(h_{1}=h_{2}=12 \mathrm{~mm}\right.$ and $t=4 \times 0.38=1.52 \mathrm{~mm})$.

In the considered examples, the shear modulus of the interlayer $G$ is varied in the range of interest for common interlayers (from $10^{-2} \mathrm{MPa}$ to $10^{1} \mathrm{MPa}$ ); consequently, the coefficient $\eta_{2 \text { Layers }}$ is calculated through (4.6), together with the deflection- and stress-effective thickness. Calculate now the value of the shear transfer coefficient $\omega$ giving the same values 
of deflection- and stress-effective thickness acording to (4.5). Note that, for a given values of the shear modulus $G$ and for a given geometry, the deflection- and stress-effective thickness of the EET approach correspond to an unique value of $\omega$.

If $\omega$ depended upon $G$ only, and if the formulation (4.5) was correct, one should find a unique correspondence between $G$ and $\omega$, independently of the problem under considerations. Figure 4.a) shows instead that, in a simply supported laminated glass beam of length $l=1.5$, for the same value of $G$ there are different values of $\omega$ depending upon the composition of the laminated package. Figure 4.b) shows that also the length of the beam $(l=1.5 \mathrm{~m}$ or $l=3 \mathrm{~m})$ is important. Therefore, the shear coupling depends not only upon the stiffeness of the interlayer, but also on the size and composition of the laminate.

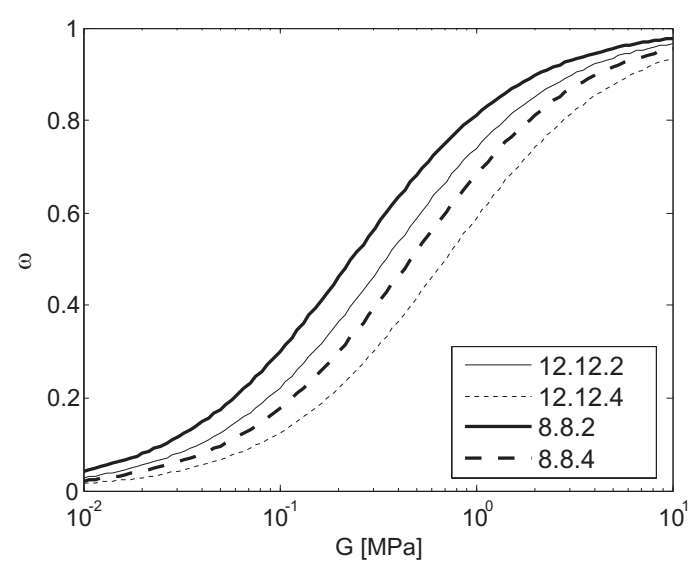

a)

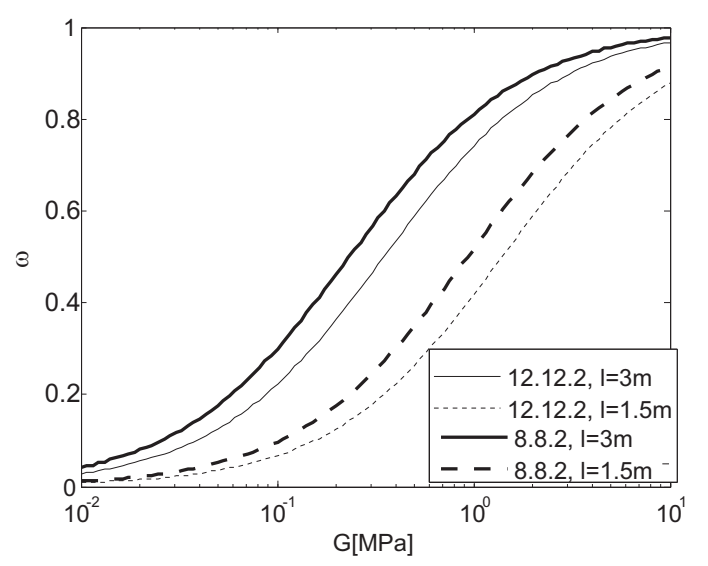

b)

Figure 4: Relationship between the shear transfer coefficient $\omega$ and the shear modulus $G$ of the interlayer for different values of beam length and thickness.

If the formulation proposed in the prEN13474 was correct, all the graphs would collapse in one curve. On the other hand, we notice noteworthy differences; for example, doubling of the length of the beam may increase $\omega$ by more than $100 \%$ (Figure $4 . b$ ). Indeed, we have verified in Sections 2 and 3 that the state of stress in a laminated glass beam strongly depends upon the length, composition, boundary and load conditions of the beam. This 
dependence is lost in the prEn13474 approach.

\subsection{Comparison with numerical experiments}

The reference example will be that of a simply supported beam of length $l=3000 \mathrm{~mm}$ and width $b=500 \mathrm{~mm}$ under uniformly distributed load $p=1 \mathrm{~N} / \mathrm{mm}$. Other loading or boundary conditions can be analyzed with the same methods used in [13]. The Young's modulus of glass plies is $E=70 \mathrm{GPa}$, while the modulus $G$ of the polymeric interlayer is varied from $10^{-2} \mathrm{MPa}$ to $10^{1} \mathrm{MPa}$, while its Poisson's ratio is supposed equal $\nu=0.49$. For the sake of comparison, two different laminated glass package are here taken into account:

- three glass plies of thickness $h_{1}=5 \mathrm{~mm}, h_{2}=8 \mathrm{~mm}, h_{3}=10 \mathrm{~mm}$, bonded by two polymeric interlayer of thickness $t_{1}=0.76 \mathrm{~mm}$ and $t_{2}=1.52 \mathrm{~mm}($ Package $A)$;

- five glass plies of the same thickness $h=6 \mathrm{~mm}$ bonded by polymeric interlayers of the same thickness $t=0.76 \mathrm{~mm}$ (Package B).

Numerical simulations have been made with the program Abaqus, using a 3-D mesh with solid 20-node quadratic bricks with reduced integration, available in the program library [19]. As shown in Fig. 5, the structured mesh has been crated by dividing the length of the beam in 100 elements, its width in 20 elements and the thickness of each glass layer in 3 elements.

The effective thicknesses obtained numerically are now compared with those obtainable with the EET method and with those calculated from the "Iterative" Wölfel- Bennison method and from the formulations of the the European Norm prEN-13474 (2012). For what the latter case is concerned, a correlation between $G$ and $\omega$ is established by calculating, for each value of $G$, the deflection-effective thickness of a two-glass-ply laminated beam with 


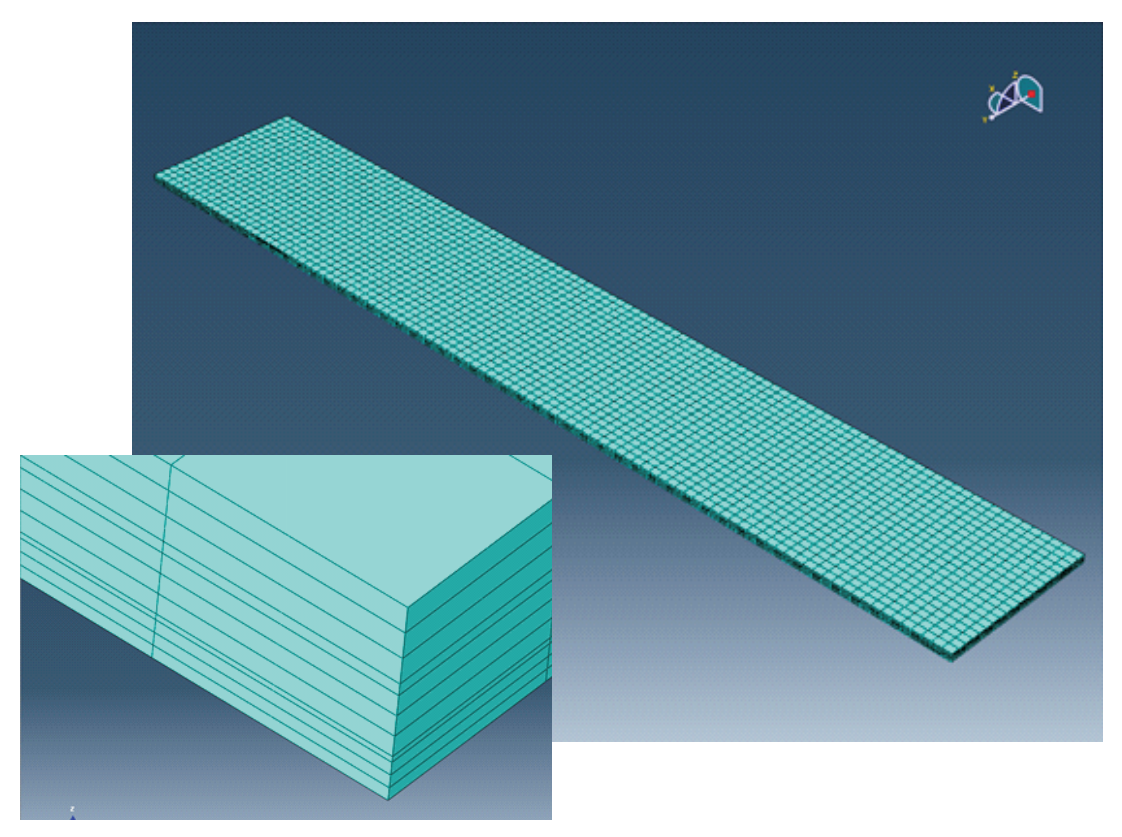

Figure 5: The mesh used in the FEM simulations for Package A.

the boundary and load conditions, and finding the value of $\omega$ that gives the same value through (4.5). It should be mentioned, however, that if the layers are not of the same thickness, the obtained value of $\omega$ depends upon which layers are chosen. Here, we have selected the two layers of the package that give the best approximation.

For Package A, Figure 6 shows the comparison of the deflection- and stress-effective thicknesses (for glass ply \# 3, the one under maximum tensile stress) obtained with the Enhanced Effective Thickness approach through (2.37) and (2.39), the iterative WölfelBennison approach, the method prescribed by prEN 13474 (equations 4.5), as well as the results of the numerical experiments. It is evident that the result achieved through EET and WB model are very accurate, whereas the prEN 13474 approach gives different results at the qualitative level. Most of all, the last method is not on the side of safeness, because it predicts deflection and stress values much lower than those predicted EET nd WB (higher effetive thicknesses). 

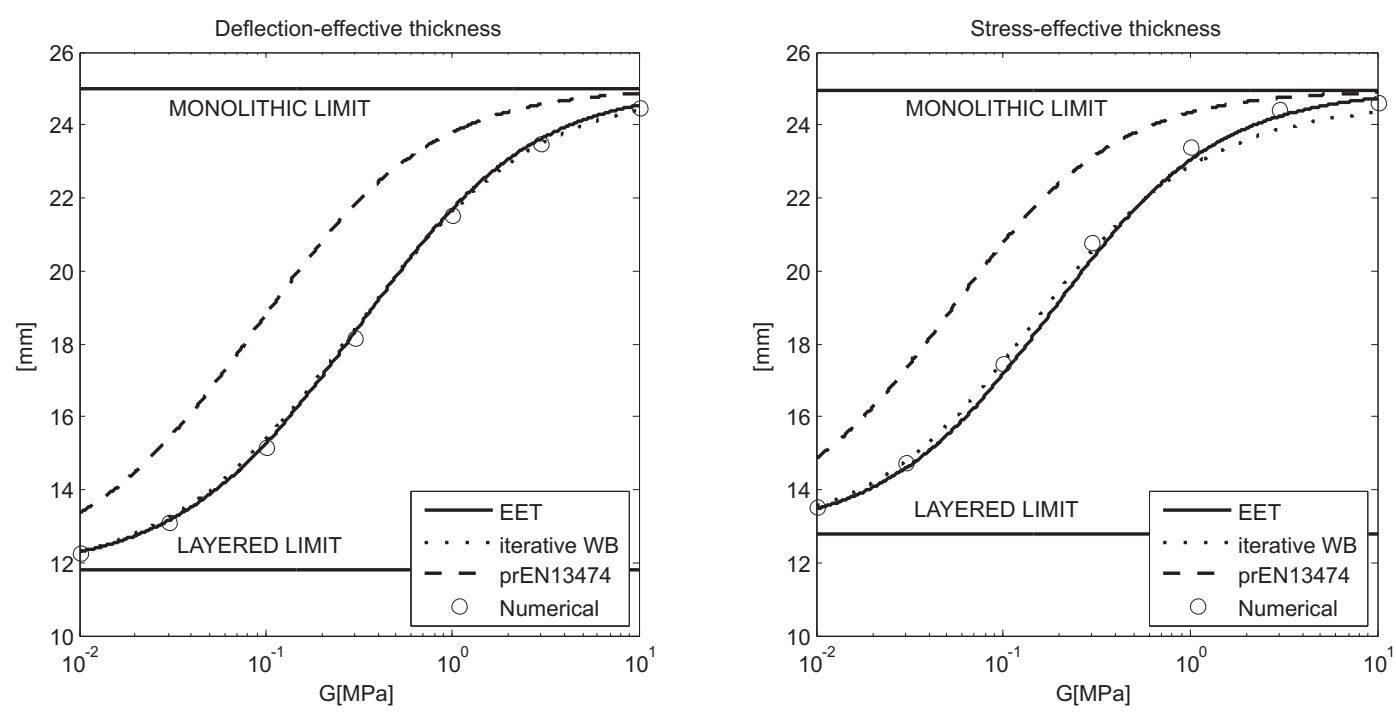

Figure 6: Simply supported beam under uniformly distributed load, Package A. Comparison of deflection- and stress-effective thicknesses obtained with: enhanced effective thickness approach (ETT); iterative Bennison-Wölfel approach (iterative WB); prEN 13474; numerical simulations.

For Package B Figure 7 compares the the deflection- and stress-effective thicknesses (of the external glass ply) obtained with the same four methods. Again, the best approximation is obtained with the EET method. The prEN13474 method is by far the less accurate.
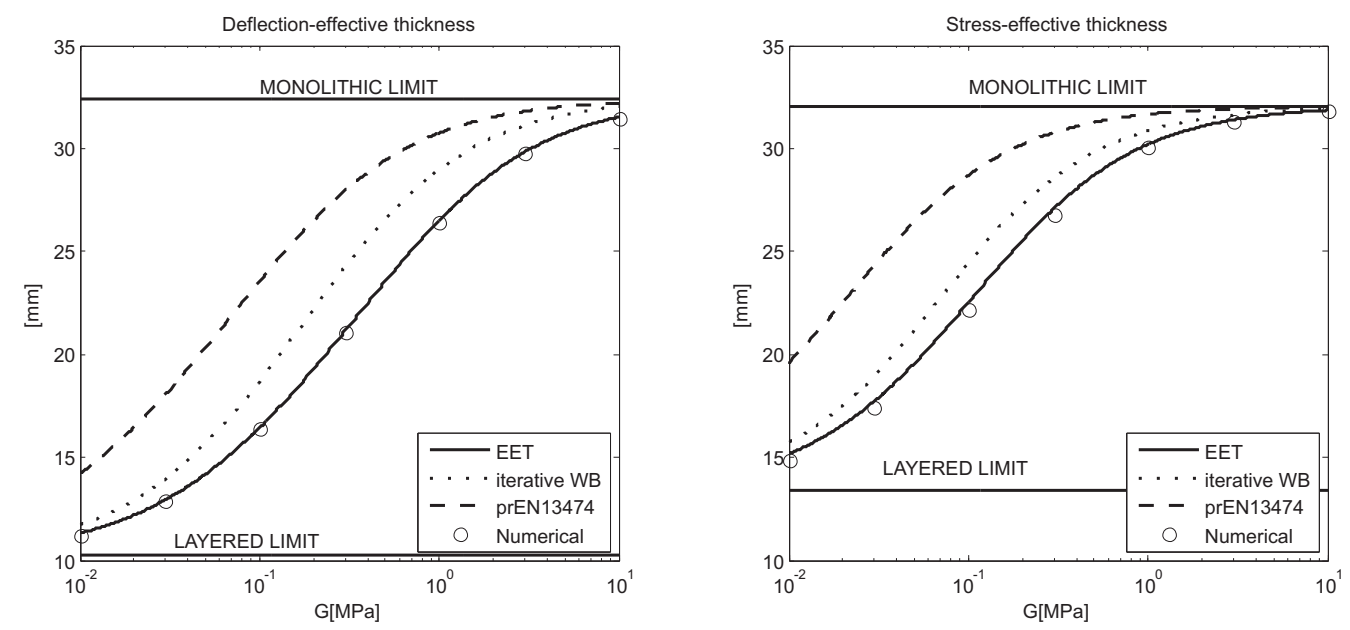

Figure 7: Simply supported beam under uniformly distributed load, Package B. Comparison of the effective thicknesses obtained with: the enhanced effective thickness approach (ETT); iterative Bennison- Wölfel approach (iterative WB); prEN 13474; the numerical simulations. 
More examples could be made by varying the load and boundary conditions of the model problem. In general, the EET approach is the one that gives the best accuracy, especially when the boundary and load conditions are different from the case of simply supported beams under uniform loading.

\section{Conclusions}

The main result of this article has been to provide a natural extension of the Enhanced Effective Thickness (EET) method proposed in [13] to the case of multi-layered laminated glass beams. The formulas (2.37) and (2.39) allow to calculate the deflection- and stresseffective stiffness for a composite package of three glass layers of any thickness with no particular difficulty. If the thickness of the glass layers is constant, the expressions (3.18) and (3.19) provide a ready-to-use practical tool for the design of laminates formed by an arbitrary number of layers. The coefficients needed for these calculations are similar to those obtained for the case of a three-layered beam (two glass layers and one interlayer) and recorded in [17]. The results that can be obtained are in excellent agreement with numerical experiments, performed with accurate three-dimensional models.

Other methods that have been proposed in technical standards give in general inaccurate results when extended to the multi-layered case. For example, the iteration of the BennisonWölfel approach [12] [18], mentioned in the ASTM standards [9], is rather complicated, provides formulas for the calculation of the state of stress in the external glass plies only (not necessarily the most stressed) and turns out to be sufficiently accurate just for simply supported beams under uniformly distributed loads. Moreover, the result depends in general upon the order in which the iteration is performed.

The formulation adopted by the project norm prEN13474 [8] is apparently very simple 
because it considers only the shear stiffness of the interlayer, but it is conceptually not justifiable. In fact, it does not take into account the important role played by load-type and size-effect (length, width and package composition) on the shear coupling that the interlayer can offer. In general, this formulation cannot be recommended in the case of three-layered beams, worst of all for multi-layered laminates.

On the contrary, the enhanced effective-thickness approach seems to represent an accurate and powerful tool for the practical calculation of laminated glass. The theory proposed here could also be applied to a package composed by an arbitrary number of layers of any thickness, even if the resulting design formulas would be slightly more complicated than those presented here. Moreover, following the same rationale, the method could be applied to the case of multilayered laminated plates, providing an extension of the theory already presented in [14].

Acknowledgements. The authors acknowledge partial support of the European Community under grant RFSR-CT-2012-00026.

\section{References}

[1] R.A. Behr, J.E. Minor, and H.S. Norville. Structural behavior of architectural laminated glass. J. Struct. Eng., 119 (1993) 202-222.

[2] J. A. Hooper. On the bending of architectural laminated glass. Int. J. Mech. Scie., 15 (1973) 309-323.

[3] H. S. Norville, K. W. King, and J. L. Swofford. Behavior and strength of laminated glass. J. Eng. Mech. - ASCE, 124 (1998) 46-53.

[4] P. Foraboschi. Behavior and Failure Strength of Laminated Glass Beams. J. Eng. Mech. - ASCE, 133 (2007) 1290-1301. 
[5] M. Asik and S. Tezcan. A mathematical model for the behavior of laminated glass beams. Comput. Struct., 83 (2005) 1742-1753.

[6] I. V. Ivanov. Analysis, modelling, and optimization of laminated glasses as plane beam. Int. J. Sol. Struct., 43 (2006) 6887-6907.

[7] P. Foraboschi. Analytical solution of two-layer beam taking into account non-linear interlayer slip. J. Eng. Mech. - ASCE, 135 (2009) 1129-1146.

[8] CEN-TC129WG8. prEN 13474: Glass in building - Determination of the strength of glass panes by calculation and testing. Project of European Stardard under Enquiry, 2012.

[9] American Society Testing of Materials. E1300-09a. Standard Practice for Determining Load Resistance of Glass in Buildings. ASTM International, 2009.

[10] S. J. Bennison and I. Stelzer. Structural Properties of Laminated Glass. In Short Course, Glass Performance Days, Tampere (Finland), 2009.

[11] S. J. Bennison, C. A. Smith, A. Van Duser, and A. Jagota. Structural performance of laminated glass made with a stiff" interlayer. In Glass Performance Days, 2001.

[12] E. Wölfel. Nachgiebiger verbund - eine näherungslösung und deren anwendungsmöglichkeiten. Stahlbau, 6 (1987) 173-180.

[13] L. Galuppi and G. Royer-Carfagni. Effective Thickness of Laminated Glass Beams. New expression via a variational approach. Eng. Struct., 38 (2012) 53-67.

[14] L. Galuppi and G. Royer-Carfagni. The effective thickness of laminated glass plates. J. Mech. Mat. Struct. , 7 (2012) 375-400.

[15] S. Timoshenko and J. N. Goodier. Theory of Elasticity. McGraw-Hill, New York, 1951. 
[16] H. Sagan. Introduction to the Calculus of Variation. Dover, New York, 1992.

[17] L. Galuppi, G. Manara, and G. Royer-Carfagni. Pratical expressions for the design of laminated glass. Comp. Part B: Eng., in press, 2012.

[18] I. Calderone, P. S. Davies, S. J. Bennison, H. Xiaokun, and L. Gang. Effective Laminate Thickness for the Design of Laminated Glass. In Glass Performance Days, 2009.

[19] ABAQUS. Analysis users manual, version 6.10. Simulia, 2010. 